\title{
Macroscopic thermoelectric efficiency of carbon nanocomposites
}

\author{
E. D. Eidelman ${ }^{1,2}$, A. P. Meilakhs ${ }^{1}$ \\ ${ }^{1} 1$ Ioffe Physical Technical Institute, 26 Politekhnicheskaya, 194021 Saint Petersburg, Russia \\ ${ }^{2}$ Saint Petersburg State Chemical-Pharmaceutical Academy, 14 Professora Popova, \\ 197376 Saint Petersburg, Russia \\ eidelman@mail.ioffe.ru,mejlaxs@mail.ioffe.ru
}

\begin{abstract}
The subject of this study is the thermoelectric efficiency $(Z)$ and the thermoelectric parameter $(Z T)$ of carbon nanocomposites, namely, the structures consisting of graphite-like $(g r)$ and diamond-like $(d)$ regions made of $s p^{2}$ and $s p^{3}$ hybridized carbon atoms, respectively. The impact of heat transfer across the boundary between $s p^{2}$ and $s p^{3}$ areas is analyzed for the first time. It is shown that the interfacial thermal resistance (Kapitza resistance) is not lower than the thermal resistance in the macroscopic $g r$ region. The influence of various factors on the Kapitza resistance is analyzed. The value of $Z T \approx 3.5$ at room temperature, taking into account the interfacial thermal resistance, is significantly higher than it would be in $g r$ films $(Z T \approx 0.75)$.
\end{abstract}

Keywords: carbon nanostructures, thermoelectricity.

Received: 28 October 2016

\section{Introduction}

In recent years, a lot of attention has been paid to the influence of size on transport phenomena in solids and, in particular, on thermoelectric effects [1-5]. Currently, attention is focused on the creation of new materials with the highest possible thermoelectric figure of merit (quality factor) $\mathrm{Z}$, which makes it possible to use this material in refrigerators or generators [2,5]: $Z=S^{2} \sigma / \chi$. Here, $S$ is the thermoelectric coefficient or the Seebeck coefficient $(\mathrm{V} / \mathrm{K}), \sigma$ is the conductivity ratio $((\mathrm{Ohm} \cdot \mathrm{m})-1)$, and $\chi$ is the thermal conductivity $(\mathrm{J} /(\mathrm{m} \cdot \mathrm{s} \cdot \mathrm{K}))$.

The higher the value of the parameter $Z T$ (where $T$ is the temperature in Kelvin) is for any material, the more useful this material is for thermoelectric conversion. Therefore, $Z T$ is used as a dimensionless quantity that characterizes thermoelectric materials.

Serious efforts were made to increase $\mathrm{Z}$, both by selecting an appropriate semiconductor and by determining its optimal layer thickness $[3,4]$. To the best of the authors' knowledge, the highest value ZT $=2.6$ at room temperature is reported in [6] for SnSe crystals.

Part of this effort is the study of thermoelectric phenomena in carbon nanostructures [3-5]. In these nanostructures, hybridized carbon atoms coexist. The coexistence of regions with very different electrical and thermal properties at such small distances from each other is a unique feature of these structures. The $g r$ areas are semimetallic with a high electrical conductivity but a relatively low thermal conductivity. The $d$ areas are wide bandgap semiconductors, dielectrics in fact, but with a high thermal conductivity. The corresponding kinetic coefficients differ in value by many orders of magnitude.

The article deals with the $Z$ values of the composite produced from $4 \mathrm{~nm}$ diamond nanoparticles generated by detonation synthesis. Such diamond nanoparticles were prepared by milling [7-9] and before long by purification techniques $[10,11]$ from agglomerates about $100 \mathrm{~nm}$ in size. Then, solid composite was produced from $4 \mathrm{~nm}$ particles by sintering at high pressure and high temperature (HPHT). Regions with $s p^{3}$ hybridization, as determined by areas of coherence, reached sizes of up to 4-12 nm. The composite consists of such particles with a $\mathrm{d}$ core, which can be covered by up to four layers of the $\mathrm{sp}^{2}$ phase, but may have no coating at all. The composite is completely free from the separate gr phase. The average particle size becomes 4-35 nm [12]. Then, the size of gr regions can be assumed at an average of $1 \approx 10-30 \mathrm{~nm}$. The thermoelectric effect and mechanisms that contribute to the increase in the thermoelectric coefficient $\mathrm{S}$, the main component of the $Z$, were discussed in $[13,14]$ for carbon nanostructures.

As for the ratio of electrical and thermal conductivity coefficients $\sigma / \chi$ being part of $Z$, it is always considered to be proportional to the temperature according to the Wiedemann - Franz law (see, e.g., [15] §78). Of course, there may be limitations associated with the possible flow of heat in the $g r$ areas. Let us further assume that the percolation threshold (see, eg, [16]) is reached [9], but the heat carried by electrons is small compared with the lattice vibrations heat transfer. 
It was recently shown that the presence of borders between the $g r$ and $d$ regions greatly influences the thermal conductivity of carbon nanostructures $[17,18]$. In this paper, we examine the effect of borders on the thermoelectric efficiency.

\section{Statement of the problem of heat transfer through the interface between phases}

Let us assume that the $g r$ area is heated or cooled on one side and contacts with a $\mathrm{d}$ area on the other side. In this case, the gr phonons migrate into $d$ or $d$ phonons migrate into gr. In accordance with Fourier's law, this heat source lowers or raises the temperature of the $g r$ lattice. The heat of the electron gas from the $g r$ area is not directly transferred to the $d$ area. However, the electrons can heat or cool the crystal lattice in $g r$. An increase in the phonon flux component takes place, and then the heat is transferred from this component to the $d$ area. This nonlinear process occurs due to the temperature difference between the electronic and phonon subsystems in $g r$. It appears as a result of presence of the boundary with $d$. The ideal structure would be the one with plane borders perpendicular to the direction of heat propagation. Any possibility of heat flow bypassing the border increases the thermal conductivity, and thus reduces the thermoelectric efficiency $Z$.

The temperature at the border undergoes a jump $\Delta T$, which is proportional to the heat flux $\mathrm{q}\left(\mathrm{W} / \mathrm{m}^{2}\right)$. Such dependence makes it possible to use the electrothermal analogy. The proportionality coefficient $r\left(\mathrm{~m}^{2} \cdot \mathrm{K} / \mathrm{W}\right)$ between the $\Delta T$ and $q$ is called interfacial thermal resistance (Kapitza resistance). The Kapitza resistance is a characteristic of the interface; in the heat transfer theory the reciprocal value of $1 / r$ is called the heat transfer coefficient.

The process being considered is a special case of heat transfer through the metal - dielectric boundary. The Kapitza resistance at the metal-dielectric interface has been widely studied (see, e.g., [17]). It was found that the Kapitza resistance, as calculated considering the nonlinearity of the process, does not depend on the dielectric characteristics and is fully determined by the properties of the metal. This thesis was experimentally confirmed in [19].

In fact, each subsystem in gr is characterized by its set of values: electron $T_{e} ; q_{e}: \chi_{e}$, and phonon $T_{p h}, q_{p h}$, $\chi_{p h}$. Each triplet of values is related according to the Fourier law.

The interaction coefficient $\theta\left(\mathrm{W} /\left(\mathrm{m}^{3} \cdot \mathrm{K}\right)\right)$ characterizes the efficiency of heat transfer between subsystems and relates the heat flux to the difference $T_{e}-T_{p h}$.

It should be noted that in experiments, the temperature is measured far away from the boundary. At this location, the temperature is $T_{e}=T_{p h}=T$, and the thermal conductivity $\chi=\chi_{e}+\chi_{p h}$ can be attributable to the general heat flow in $g r: q=q_{e}+q_{p h}$. In $g r$, it can be assumed that $\chi_{e}>>\chi_{p h}$ and heat transfer through the electronic subsystem dominates.

Only phonons can pass from $g r$ to $\mathrm{d}$, hence $q_{p h}=q ; q_{e}=0$ at the boundary.

According to [19], the problem is posed so that the properties of $d$ do not affect the $T_{e}$ and $T_{p h}$ temperature in $g r$.

\section{Temperature and thermal resistance alignment at the boundary between $g r$ and $d$}

The solution of the problem is completely similar to that provided in [17]. The result is that the characteristic distance $\lambda$, at which the temperatures of the electron and phonon subsystems in $g r$ are actually aligned, is related to the system characteristics as:

$$
\lambda=\sqrt{\frac{\chi_{e} \chi_{p h}}{\theta \chi}} \simeq\left(\chi_{p h} / \theta\right)^{1 / 2} .
$$

The components of the thermal resistance are determined by the jumps of the subsystems temperatures, $T_{e}(0)$ and $T_{p h}(0)$, relative to the temperature $T_{d}$ in $d$, which is equal to $T_{d}(0)$ at the boundary (Fig. 1 ). In the course of the experiment, the temperature gradient from a region far from the boundary is usually extrapolated linearly to the boundary. This temperature $T_{g r}$ is considered to be the $g r$ temperature; accordingly, it is $T_{g r}(0)$ at the border, and the temperature jump is considered equal to $\Delta T=T_{g r}(0)-T_{d}(0)$. However, almost always $T_{g r}(0) \approx T_{e}(0)$.

The Kapitza thermal resistance caused by the measured jump $\Delta \mathrm{T}$ can be divided into $r_{p h}$, arising due to the temperature jump $\Delta \mathrm{T}_{p h}$ of phonons in $g r$ and $d$ areas, and $r_{r}$, arising because the electronic and phonon subsystems in gr are heated unequally near the border. Therefore, $r=r_{p h}+r_{r}$.

The thermal resistance (relative!) $r_{r}$ arises due to the difference in temperature jumps of the electron $\Delta T_{e}$ and phonon $\Delta T_{p h}$ subsystems: $\Delta T_{r}=T_{e}-T_{p h}=\Delta T_{e}-\Delta T_{p h}$. In practice, the temperature in $g r$ is determined by the electronic subsystem temperature. So, the measured jump $\Delta T \approx \Delta T_{e}$ and $\Delta T_{r}$ can be calculated as $\Delta T_{r}=\Delta T-\Delta T_{p h}$. 


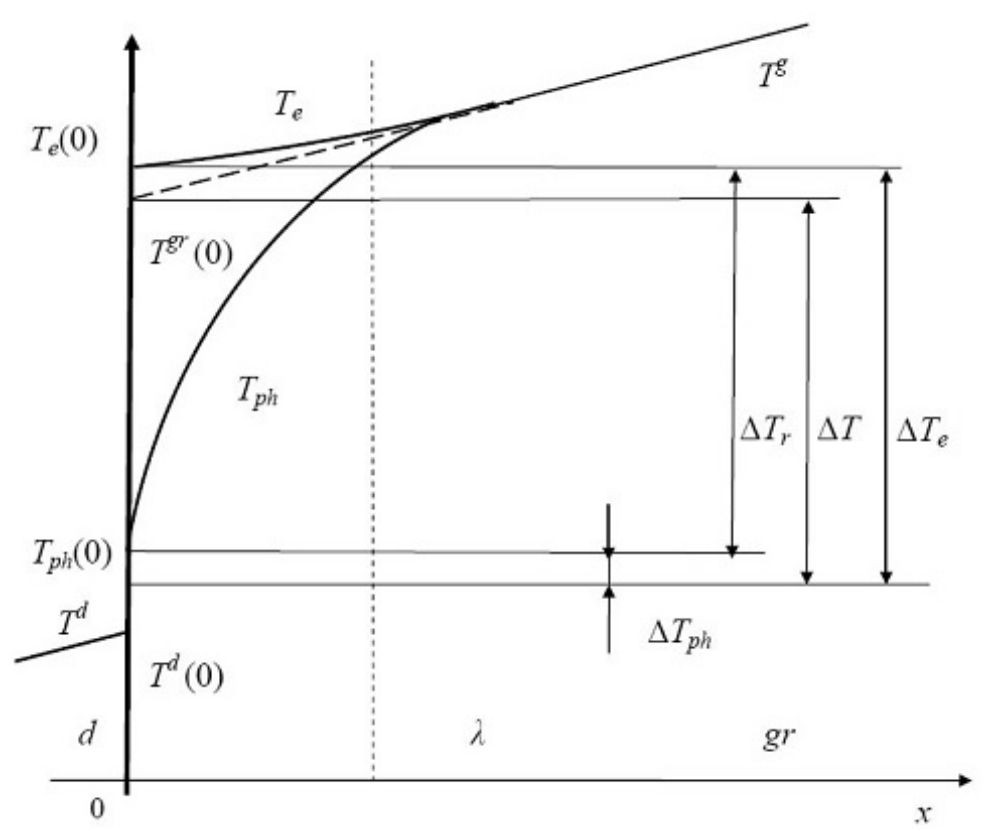

FIG. 1. This figure presents the temperatures in the graphite-like $(g r)$ area $T_{g r}$ (on the right) and the diamond-like $(d)$ area $T_{d}$ (on the left), near the boundary located at $x=0$. At the boundary, the temperatures are, respectively, $T_{g r}(0), T_{d}(0), T_{e}(0)$ and $T_{p h}(0)$. Also shown are values of the corresponding temperature jumps $\Delta T_{p h}$ and $\Delta T_{e}$ at the border to the temperature $T^{d}(0)$. The jump $\Delta T=\operatorname{Tgr}(0)-T^{d}(0)$ is measured experimentally. Finally, the temperature differences in $g r$ subsystems were shown to be caused by the Kapitza resistance due to the presence of the boundary $\Delta T_{r}$

The value of the jump $\Delta T_{p h}$ determines the proportion of phonons reflected from the boundary back into $g r$. This proportion was determined in [13] for a system very similar to the carbon nanocomposites under study. There, it was found to be usually small. Therefore, $\Delta T_{p h}<<\Delta T_{r}$ and accordingly $r_{p h}<<r_{r}$. This inequality is the major, roughest approximation that is used for the solution of the problem. The values in Fig. 1 are provided using this approximation.

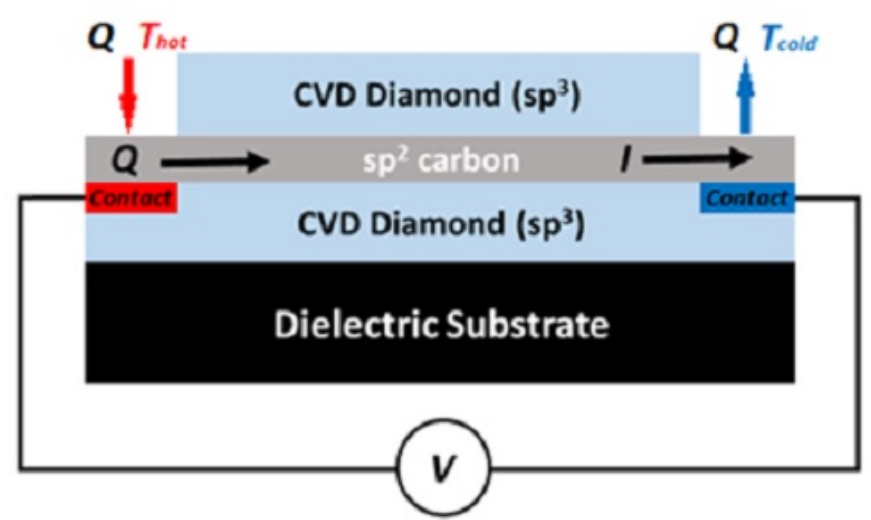

FIG. 2. A possible thermoelement design, where the heat $Q$ and the temperature difference $T_{\text {hot }}-T_{\text {cold }}$ provide the conditions (see [13]) under which the quasi-ballistic drag of electrons by phonons prevails in the carbon nanostructure

Next, it will be assumed that the Kapitza resistance in carbon nanostructures and, in particular, carbon nanocomposites produced by HPHT, is determined by the relative temperature jump, which is approximately equal to the measured temperature jump $\Delta T_{r} \approx \Delta T$, and therefore, the thermal resistance is determined approximately by the relative thermal resistance $r \approx r_{r}$. 
Then the solution obtained in [17] can be written for the simplest one-dimensional heat propagation as:

Then the thermal resistance:

$$
\begin{aligned}
& T^{g r}-T_{e}=\frac{1}{\chi} q x+\frac{\lambda q}{\chi} e^{-x / \lambda} \\
& T^{g r}-T_{p h}=\frac{1}{\chi} q x-\frac{\lambda q}{\chi} \frac{\chi_{e}}{\chi_{p h}} e^{-x / \lambda} .
\end{aligned}
$$

$$
r \simeq=r_{r}=\frac{\lambda \chi_{e}}{\chi \chi_{p h}} \approx \frac{1}{\sqrt{\theta \chi_{p h}}}
$$

is expressed in terms of the thermal conductivity of the phonon subsystem in gr and heat transfer efficiency between the electron and phonon subsystems in the same area.

\section{Thermoelectric efficiency}

The thermoelectric efficiency $Z_{b}$ of the system at the boundary between $g r$ and $d$ can be represented now as:

$$
Z_{b}=\frac{S^{2} \sigma}{\chi_{e f}} \approx S^{2} \sigma\left(\frac{1}{\chi}+\frac{r}{l}\right) .
$$

This formula is stated using the electrothermal analogy. The magnitude of the thermoelectric efficiency is expressed through the effective thermal conductivity $\chi_{e f}$ of the system that takes into account the thermal conductivity in the $g r$ area with the overall size of 1 , and the thermal resistance of the boundary.

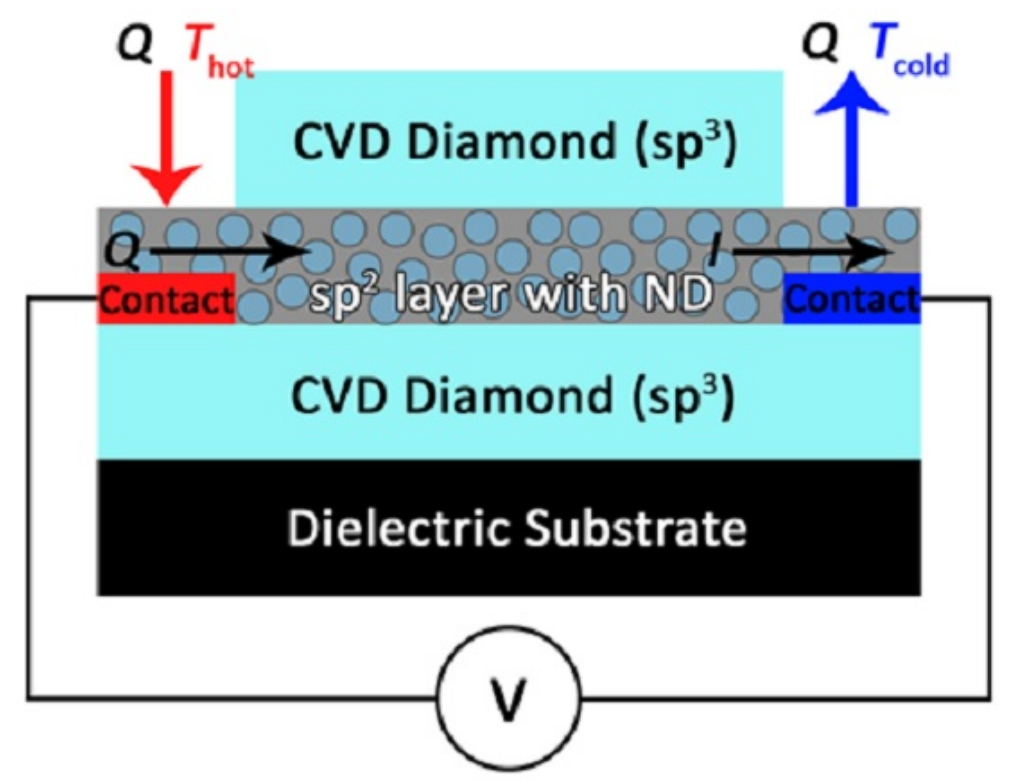

FIG. 3. A possible thermoelement design. In addition to the conditions corresponding to the structure shown in Fig. 2, the Wiedemann - Franz law is violated because of the introduction of diamond nanoparticles (ND). The thermal conductivity is determined by the Kapitza thermal resistance, i.e. by heat transfer at the interface of $s p^{2} / s p^{3}$ areas

Of course, even for macroscopic and microscopic samples, this formula provides the value $\mathrm{Z}$ mentioned in the Introduction. For small values of $1<<\lambda$ in nanosystems, the addition generated by a decrease in scattering due to heat transfer from the electron subsystem to the phonon subsystem, can significantly increase the thermoelectric efficiency.

Let us estimate, first of all, the value of $\theta$, the efficiency coefficient of heat transfer between the subsystems. As the primary heat flux is carried by electrons, we can assume that energy is transmitted from them to the crystal lattice. It is clear that energy can be transmitted by quanta only and the characteristic energy of the quanta is determined by the temperature of phonons: $T_{p h}$ when $T_{p h}<T_{D}$, or Debye temperature $T_{D}$ in case $T_{p h}>T_{D}$. Of course, the temperature difference between the subsystems is small compared with each subsystem temperature, it may be a few tenths of a degree or a few degrees, but the heat transfer occurs with the phonon transition. The phonons have the specified temperature $T_{p h} \approx T_{D}$ in the $s p^{2}$ region. On the other hand, the gradient of the thermal 
flow of electrons is expressed in terms of the heat generated by the current, which would correspond to this heat flux. The result is:

$$
\theta=a \frac{c^{2} e^{2} n_{0}^{2}}{\sigma T_{D}}
$$

Here, $\mathrm{a}$ is the order of unity factor, $\mathrm{c} \approx 1.5 \cdot 10^{3} \mathrm{~m} / \mathrm{s}$ is the speed at which the electrons move, because they captivate phonons, $n_{0} \approx 10^{25} \mathrm{~m}^{-3}$ is the conduction electron density, $\sigma \approx 10^{3}(\mathrm{Om} \cdot \mathrm{m})^{-1}$ is the conductivity coefficient, $T_{D} \approx 0.5 \cdot 10^{3} \mathrm{~K}$, as already indicated, is the $s p^{2}$ region Debye temperature, and $e=1.6 \cdot 10^{-19} \mathrm{Cl}$ is the electron charge. The values of all variables, except, of course, the universal value of the electron charge, are given for graphite. All values are weakly dependent on the temperature and are borrowed from [20]. By substituting these values, we get $\theta \approx 10^{15} \mathrm{~W} /\left(\mathrm{m}^{3} \mathrm{~K}\right)$. Apparently, smaller values should be selected in reality. Of course, this assessment is by the order of magnitude. Tab. 1).

In [21] and [22] the following values of graphite thermal conductivity versus temperature are given (see.

TABLE 1. Graphite thermal conductivity versus temperature

\begin{tabular}{|c|c|c|c|c|c|c|c|c|}
\hline$\chi_{g r}$ & $\mathrm{Bt} /(\mathrm{m} \cdot \mathrm{K})$ & 114 & 86 & 61 & 47 & 40 & 34 & 25 \\
\hline$T$ & $\mathrm{~K}$ & 293 & 473 & 873 & 1173 & 1473 & 1774 & 2073 \\
\hline
\end{tabular}

Data for diamond $\chi_{d}=1000 \mathrm{~W} /(\mathrm{m} \cdot \mathrm{K})$ at $\mathrm{T}=273$ and $42 \mathrm{~W} /(\mathrm{m} \cdot \mathrm{K})$ at $4323 \mathrm{~K}$ are also provided. It is obvious that the thermal conductivity for $g r$ must be somewhat intermediate. In [23,24] $\chi_{g r}$ values between 200 and $300 \mathrm{~W} /(\mathrm{m} \cdot \mathrm{K})$ are provided. On the other hand, the phonon part of thermal conductivity accounts for a small part of the total value. For further estimates, $\chi_{p h} \approx 5-10 \mathrm{~W} /(\mathrm{m} \cdot \mathrm{K})$ will be taken (see [25]).

According to the formula (1) it turns out that $\lambda \approx 100 \mathrm{~nm}$, which confirms the above assumption on the size ratio of $g r$ and the characteristic distance at which the temperatures of the electron and phonon subsystems can be regarded as being equal. Thus, the thermal conductivity in the carbon nanocomposites is no longer dependent on the size of the $g r$ area, just like in the case where the interface is less than $4 \mathrm{~nm}$ thick (for oxides) the thermal conductivity is no longer dependent on the layer thickness [26-28].

Now, using the formulas (5) and (3) it is easy to estimate the thermal resistance. We obtain $r \approx 10^{-9} \mathrm{~m}^{2} \mathrm{~K} / \mathrm{W}$. The same value of the thermal resistance is derived with an entirely different reasoning in [24]. Finally, let us find the relative thermoelectric efficiency:

$$
\frac{Z_{e f}}{Z}-1=\frac{r \chi^{g r}}{l}
$$

As stated above (see Introduction), we can assume that if the size of the graphite-like area is on the order of $1 \approx 10-30 \mathrm{~nm}$, it turns out that the thermoelectric efficiency of carbon nanocomposites is ten times higher than that of graphite. At room temperature, the thermoelectric parameter is 5-20 times higher than that of graphite.

\section{Conclusion}

As is known (see, for example, [20]), the Seebeck coefficient of graphite depends on its purity, but it is of the order of $S=2-3 \mu \mathrm{V} / \mathrm{K}$. Thus, the graphite-like layer, whose surfaces have the temperature difference $100 \mathrm{~K}$, produces the voltage of $0.3 \mathrm{mV}$. This value does not depend on the layer thickness, but the layer geometry affects the conditions of heating and heat dissipation and, consequently, the thermoelectric efficiency. At the surface with the lower temperature of $300 \mathrm{~K}$, subject to the Wiedemann - Franz law, such a thermoelement would have the thermoelectric parameter $Z T=3 \cdot 10^{-4}$. Materials from carbon nanostructures have $S=10-20 \mu \mathrm{V} / \mathrm{K}[28]$ and they could produce the voltage of $1-2 \mathrm{mV}$ with $Z T=3 \cdot 10^{-2}$ with the same temperature differences. However, if such conditions are provided under which the quasi-ballistic drag of electrons by phonons prevails in the carbon nanostructure [13], it would be possible to obtain $5-10 \mathrm{mV}$ and $Z T=0.75$ (Fig. 2). In nanocomposites with the quasi-ballistic drag, in which the Wiedemann - Franz law is not fulfilled, namely, the thermal conductivity is determined by the Kapitza thermal resistance, it is possible to achieve the thermoelectric parameter 3.5 (Fig. 3).

\section{Acknowledgement}

The authors are grateful to Professor Dieter Gruen who pointed to the existence of the efficiency problem for the engineering of generators and refrigerators operating on the thermoelectric effect. We would like to acknowledge A.Ya. Vul' for his attention, which enabled us to complete this work. We are also thankful to F.M. Shakhov for fruitful discussions and the design of the figures. This work was supported by RSF grant No. 16-19-00075. 


\section{References}

[1] Heremans J.P. Thermoelectricity: The ugly duckling. Nature, 2014, 508, P. 327-328.

[2] Enhanced thermoelectric power in bismuth nanocomposites. Patent 6670.539 United States. Joseph Pierre Heremans, Cristofer Mark Thrush, Donald T. Morecli, 2003.

[3] Wang Y., Jaiswal M., Lin M., Saha S., Ozyilmaz B., Loh K.P. Electronic properties of nanodiamond decorated graphene. ACS Nana, 2012, 6(2), P. 1018-1025.

[4] Vul' A., Reich K., Eidelman E., Terranova M.L., Ciorba A., Orlanducci S., Sessa V., Rossi M. A Model of Field Emission from Carbon Nanotubes Decorated by Nanodiamons. Advanced Science Letters, 2010, 3, P. 1-8.

[5] The thermoelectric element. Invention. Patent 2,376,681 Russia. Vul A.Ya., Eidelman E.D. The legal owner of Physics - Technical Institute. AF Ioffe RAS, 2008.

[6] Zhao L.-D., Lo S-H., Zhang Y., Sun H., Tan G., Uher C., Wolverton C., Dravid V.P., Kanatzidis M.G. Ultralow thermal conductivity and high thermoelectric figure of merit in SnSe crystals. Nature, 2013, 508, P. 373-377.

[7] Krueger A., Kataoka F., Ozawa M., Fujino T., Suzuki Y., Aleksenskii A.E., Vul'A.Ya., Osawa E. Unusually tight aggregation in detonation nanodiamond: identification and disintegration. Carbon, 2005, 43, P. 1722-1726.

[8] Eydelman E.D., Siklitsky V.I., Sharonova L.V., Yagovkina M.A., Vul' A.Ya., Takahashi M., Inakuma M., Ozawa M., Osawa E. A stable suspension of single ultrananocrystalline diamond particles. Diamond and Related Materials, 2005, 14, P. 1765-1769.

[9] Vul' A.Ya., Eidelman E.D., Inakuma M., Osawa E. Correlation between viscosity and absoption of electromagnetic waves in an aqueous UNCD suspension. Diamond and Related Materials, 2007, 16, P. 2023-2028.

[10] Williams O., Hees A., Dieker C., Jager W., Kirste L, Nebel C. Size-dependent reactivity of diamond nanoparticles. ACS Nano, 2010, 4, P. 4824-4830.

[11] Aleksenskii A., Eydelman E., Vul' A.Ya. Deaglomeration of detonation nanodiamonds. Nanosci. Nanotechnol. Lett., 2011,3 , P. 68-74.

[12] Kidalov S.V., Shakhov F.M., Lebedev V.T., Orlova D.N., Grushko Y.S. Small-angle neutron scattering study of high-pressure sintered detonation nanodiamonds. Crystallogr. Rep., 2011, 56(7), P. 1181-1185.

[13] Eidelman E., Vul' A.Ya. The strong thermoelectric effect in nanocarbon generated by the ballistic phonon drag of electrons. Journal of Physics: Condensed Matter, 2007, 19, P. 1-8.

[14] Koniakhin S.V., Eidelman E.D. Phonon drag thermopower in graphene in equipartition regime. EuroPhysLett., 2013, 103(8), P. 1-6.

[15] Lifshitz E.M., Pitaevskii L.P. Physical kinetics. Pergamon International Library of Science, Technology, Engineering and Social Studies. 2008, $482 \mathrm{p}$.

[16] Efros A.L. Physics and Geometry of Disorder. Percolation Theory. Imported Pubn, 2004, 256 p.

[17] Meilakhs A.P., Eidelman E.D. Overheating or overcooling of electrons in a metal because of the effect of an interface with an insulator. JETP. Lett., 2014, 100(2), P. 81-85.

[18] Eidelman E.D. Estimation of the contact area of solids by electrothermal analogy. Nanosystems: Physics, Chemistry, Mathematics, 2015, 6(4), P. 547-550.

[19] Costescu R.M., Wall M.A., Cahill D.G. Thermal conductivity of thin films. Measurements and understanding. Phys. Rev B., 2003, 67, P. 054302-1-8.

[20] Prut V.V., IFE Working Paper, 2007, 30, P. 6462.

[21] Properties and Characteristics of Graphite. Entegris, Inc., 2013, 38 p.

[22] Levinshtein M., Rumyantsev S., Shur M. Handbook series on Semiconductor Parameters. World Scientific, Singapore, 2007,219 p.

[23] Kidalov S.V., Shakhov F.M., Vul' A.Ya. Thermal conductivity of nanocomposites based on diamonds nanodiamonds. Diamond and Related Materials, 2007, 17, P. 844-847.

[24] Kidalov S.V., Shakhov F.M., Vul' A.Ya. Thermal conductivity of sintered nanodiamonds and microdiamonds. Diamond and Related Materials, 2008, 17, P. 844-847.

[25] Kidalov S.V., Shakhov F.M., Vul' A.Ya., Ozerin A.N. Grain-boundary heat conductance in nanodiamond composites. Diamond and Related Materials, 2010, 19, P. 976-980.

[26] Cahill D.G., Ford W.K., Goodson K.E., Mahan G.D., Majumdar A., Maris H.J., Merlin R., Phillpot S. R. Nanoscale thermal transport. Journal of Applied Physics, 2003, 93, P. 793-798.

[27] Losego M.D., Grady M.E., Sottos N.R., Cahill D.G., Braun P.V. Effects of chemical bonding on heat transport across interfaces. Nature materials, 2012, 11, P. 502-506.

[28] Cahill D.G., Braun P.V., Chen G., Clarke D.R., Fan S., Goodson K.E., Keblinski P., King W.P., Mahan G.D., Majumdar A., Maris H.J., Phillpot S.R., Pop E., Shi L. Nanoscale thermal transport. II. 2003-2012. Applied physics reviews, 2014, 1, P. 011305-1-45.

[29] Zolotukhin I.V., Golev I.M., Markova A.E., Panin Yu.V., Sokolov Yu.V., Tkachev A.G., Negrov V.L. Some properties of solid fractal structures in carbon nanofibers. Technical Physics Letters, 2006, 32(3), P. 199-200. 